\title{
Frequency adaptive Sliding Fourier Transform for synchronizing VSI to the grid
}

Osama M. Arafa, M. E. Abdallah, Ghada A. Abdel Aziz

Department of Power Electronics and Energy Conversion, Electronics Research Institute, Egypt

\begin{tabular}{|c|c|}
\hline Article Info & ABSTRACT \\
\hline Article history: & \multirow{13}{*}{$\begin{array}{l}\text { Sliding Discrete Fourier Transform (SDFT) is very efficient regarding } \\
\text { computational load and it possesses a very fast phase angle detection with } \\
\text { excellent harmonic rejection at nominal frequency. However, at off-nominal } \\
\text { frequency, SDFT generates errors in both magnitude and phase angle due to } \\
\text { spectral leakage. This paper introduces a workaround for Fourier Transform } \\
\text { to handle this disability under off-nominal frequency while avoiding } \\
\text { variable-rate sampling. Sliding Fourier Transform (SFT) is used as a phase } \\
\text { detector for a phase-locked loop whose output frequency is used to drive the } \\
\text { SFT. The paper revisits the mathematics of Fourier Transform (FT) in a } \\
\text { three-phase setting via a time-domain approach to show a newly proposed } \\
\text { filtering technique for the double-frequency oscillation just by summing the } \\
\text { FT sine/cosine filter outputs of the three individual phases. Also, the analysis } \\
\text { aims to determine and correct the phase and magnitude errors under off- } \\
\text { nominal frequency operation. The proposed technique (SFT-PLL) is tested in } \\
\text { real time on dSPACE DS1202 DSP using voltage vectors that are pre- } \\
\text { generated to simulate the most adverse grid conditions. The testing scenarios } \\
\text { compare the performance of the SFT-PLL with that of the Decoupled } \\
\text { Stationary Reference Frame PLL (d } \alpha \beta \text { PLL). The results prove that SFT-PLL } \\
\text { is superior to d } \alpha \beta \text { PLL. }\end{array}$} \\
\hline Received Dec 19, 2018 & \\
\hline Revised Feb 20, 2019 & \\
\hline Accepted Mar 8, 2019 & \\
\hline Keywords: & \\
\hline Discrete fourier transform & \\
\hline Fourier transform & \\
\hline Renewable energy sources & \\
\hline & \\
\hline Synchronization & \\
\hline & \\
\hline & \\
\hline & \\
\hline
\end{tabular}

Copyright (C) 2019 Institute of Advanced Engineering and Science. All rights reserved.

\section{Corresponding Author:}

Osama M. Arafa,

Department of Power Electronics and Energy Conversion,

Electronics Research Institute, Dokki, Egypt.

Email: oarafa2004@yahoo.com

\section{INTRODUCTION}

Fuel cells and other Renewable Energy Sources (RES), such as PV systems and Wind Energy Converters (WECS), use inverters to inject the captured energy into the utility grid [1]. The successful integration of these sources to the electrical grid is still having a lot of issues and technical challenges [1]. One of the most critical issues of these integrations is the synchronization of the grid-side converter (GSC) to the electrical grid [2]. The synchronization of the GSC to the electrical grid can be defined as the process of minimizing the difference in phase angle, frequency and voltage between the GSC output and the utility grid waveform [3]. This synchronization process has to be realized before the connection between the RES or the GSC and the utility grid takes place, then it is to be maintained after the connection is established.

In [4], the ideal synchronization technique must instantly respond to any grid changes, efficiently track the phase angle of the electrical grid, competently detect any variation in the grid frequency and competently eliminate harmonic components and disturbance from the fundamental waveform. There are lots of methods used for tracking the phase angle of the grid voltage such as zero-crossing detection method (ZCD), Space Vector (SV) method, Kalman filter, phase-locked loop methods (PLL) and Discrete Fourier Transform (DFT). The problem is none of these methods completely satisfies the full requirements of an ideal synchronization technique 
Zero-Crossing Detection (ZCD) is the simplest angle synchronization method. The zero-crossing point is detected twice every single cycle and the interval between each two successive crossings is measured to any desired resolution. This method is very simple but it can be used only if the input signal is a stable sinusoidal signal as it is very sensitive to harmonics and noise which can cause multiple contiguous zero crossings [5].

Phase locked loop is a well-established technique and there are a lot of implementations of PLL synchronization. Each implementation differs from the other from the point of view of simplicity, immunity to disturbance, robustness in case of grid faults, fast convergence to the grid angle, and how it deals with disturbances. Synchronous Frame PLL (SF-PLL) technique is widely used in three-phase grid-connected power converters for its simple implementation, fast and accurate estimation of the phase angle of the grid [6]. Under ideal grid conditions, without any disturbances or harmonics, this technique exhibits fast-tracking. However, it is not a good solution in the presence of unbalanced grid voltages [7].

Real and imaginary power phase-locked loop (PQ-PLL) is a robust digital PLL algorithm based on the instantaneous real and imaginary power. This algorithm is introduced to maintain synchronization in presence of harmonics and negative sequence unbalances. Another algorithm which can deal with unbalanced grid voltages is the Double Synchronous Frame PLL (DSF-PLL). It is a very suitable solution for gridconnected converters that operate in unstable frequency grid and unbalanced conditions [8].

Sinusoidal signal integrator PLL algorithm (SSI-PLL) has the advantage of operating well under unbalanced and distorted grid [8]. Another advantage of this algorithm is that it can be used for the singlephase system with a small modification. Enhanced phase-locked loop (EPLL) is a frequency-adaptive nonlinear technique. EPLL shows a high degree of robustness to the harmonic, noise, and unbalanced grid [9]. This algorithm can be used for single phase applications. Quadrature PLL (QPLL) is based on estimating the in-phase and the quadrature phase value of the fundamental component of the input voltage signal. This method is a good solution for distributed generation [10]. Predictive Phase-Locked Loop (PPLL) is a complex synchronization method. However, PPLL has a wide-range synchronization capability and the synchronization information can be taken out within two voltage cycles under the worst case. Stationary Frame PLL $(\alpha \beta$ PLL) in [11], Decoupled Double Synchronous Reference Frame PLL (ddsrfPLL) and Decoupled Stationary Reference Frame (d $\alpha \beta P L L)$ techniques have the widest use in industry. The limitation of the $\alpha \beta P L L$ is its disability to track the grid angle in the case of unbalanced faults. The ddsrfPLL technique overcomes this limitation by decoupling the negative and the positive sequence of the grid voltage but the severe drawback of this technique is the high overshoot in the estimated grid angle when the fault occurs. The d $\alpha \beta$ PLL overcomes this drawback but it spends more than two cycles to track the grid frequency in case of frequency change.

Discrete Fourier transform method (DFT) is one of the earliest methods used for phase angle estimation. This method provides a high degree of immunity against harmonics [12]. Sliding Discrete Fourier Transform (SDFT) is an improved version of the DFT in which optimized use of computation power and reduced latency are provided by using a recursive formulation of the DFT algorithm. However, if the time window of the SDFT is not identical with the grid period, a phase shift occurs between the estimated angle and the actual grid angle. In [12] some strategies have been reported to compensate for this phase error such as the use of time-varying Fourier coefficients and polynomial regression analysis. In fact, these strategies are rendered with noticeable complexity. Other fixing methods for the SDFT drawback are presented in [13]. However, the presented methods involve the use of a PI controller and a Numerically Controlled Oscillator (NCO). The method adopts a variable sampling rate of the grid voltage controlled by the NCO. Variable rate sampling adds difficulty to embedding the SDFT algorithm in the main application DSP controller which normally runs at fixed execution rate. Although the two proposed methods in [13] are simpler than those of [10], both methods compensate the phase error on a cycle-by-cycle basis, which is rather a slow process. The undesirable features of the SDFT in the case of off-nominal frequency are not only limited to the phase error which is a function of the frequency deviation but also includes a high-frequency oscillation observable in the magnitude and phase angle estimates. This high-frequency oscillation is referred to in the literature as double-frequency ripple and some post-processing for frequency and phase angle estimates in the offnominal operation mode are proposed. The post-processing can be done either by simple digital averaging filter or by resampling filter which is relatively more complex in implementation.

From the previous survey, it appears that there is still some room for improvement in the phase angle tracking issue especially in the case of variable and off-nominal grid frequency accompanied by high harmonic content and/or in unbalanced three-phase systems. This paper revisits the FT concept through a time-domain analysis to further analyze the undesirable features associated with the off-nominal grid frequency. An efficient and natural filtering method for the double-frequency oscillations is devised. The laws relating the phase and magnitude errors to the frequency difference are derived. The formulation of the 
FT in a sliding mode is explained. Experimental testing is used to confirm the validity of the proposed techniques.

This paper is organized as follows: the mathematical analysis for determining the phase and magnitude errors under off-nominal frequency condition is presented in section 2 . The recursive implementation is explained in section 3. The structure of the proposed SFT-PLL is explained in section 4. The method used for experimental testing and the test results in real-time on a DSP prototyping platform (DS1202 by dSPACE ${ }^{\mathrm{TM}}$ ) are presented in sections 4 and 5, respectively. Section 6 presents the conclusions of this paper.

\section{MATHEMATICAL ANALYSIS}

The grid voltages are represented by a balanced set of three-phase sinusoidal voltage $V_{g}$ with a frequency $f_{g}(\mathrm{~Hz})$ and with a peak value $E$ (Volt), thus $V_{g}$ is given by:

$$
\begin{aligned}
& V_{g a}=E \sin \left(2 \pi f_{g} t+\theta_{0}\right), \\
& V_{g b}=E \sin \left(2 \pi f_{g} t-\frac{2 \pi}{3}+\theta_{0}\right) \\
& V_{g c}=E \sin \left(2 \pi f_{g} t+2 \pi / 3+\theta_{0}\right)
\end{aligned}
$$

where $\theta_{0}$ is the initial phase shift (rad) of the voltage $V_{g a}$, this phase shift depends on the random instant at which the measurement is started $(t=0)$. For the sake of this analysis $f_{g}$ will be assumed constant.

The Fourier transform is based on applying two orthogonal filters, namely a sine filter and a cosine filter on the input signal. The point here is that we set the phase sequence and the phase shift between the sine/cosine filters in a way that serve additional filtering purpose as will be seen later on. Therefore, the three grid voltages will be processed using two sets of balanced three-phase signals $V_{c x}$ and $V_{c y}$ having the same phase sequence as the grid voltage, running at a pre-known nominal grid frequency $f_{n}(\mathrm{~Hz})$ and having a unity peak value. For the sake of this analysis $f_{n}$ will be assumed constant [14].

The first set of signals $V_{c x}$ is given by sine functions and is called the sine or direct set:

$$
\begin{aligned}
& V_{c a x}=\sin \left(2 \pi f_{n} t\right), \\
& V_{c b x}=\sin \left(2 \pi f_{n} t-\frac{2 \pi}{3}\right) \\
& V_{c c x}=\sin \left(2 \pi f_{n} t+2 \pi / 3\right)
\end{aligned}
$$

The second set of voltages $V_{c y}$ is given by cosine functions and is called the cosine or quadratic set:

$$
\begin{aligned}
& V_{c a y}=\cos \left(2 \pi f_{n} t\right), \\
& V_{c b y}=\cos \left(2 \pi f_{n} t-\frac{2 \pi}{3}\right) \\
& V_{c c y}=\cos \left(2 \pi f_{n} t+2 \pi / 3\right)
\end{aligned}
$$

The Fourier transform requires performing the following integrations to get the angle $\theta_{0}$ :

$$
\begin{aligned}
& x_{i}=\frac{1}{T_{n}} \int_{t}^{t+T_{n}} v_{c i x} \cdot v_{g i} d t \\
& y_{i}=\frac{1}{T_{n}} \int_{t}^{t+T_{n}} v_{\text {ciy }} \cdot v_{g i} d t
\end{aligned}
$$

(4) and (5) are compact forms for the three-phase where the subscript $i=\mathrm{a}, \mathrm{b}, \mathrm{c}$ and $T_{n}=1 / f_{n}$. For any positive and non-zero value of $f_{n}$, two cases are considered for calculating these integrals, the first case is $f_{g} \neq f_{n}$ (off-nominal frequency case) which is the generic case and the second case $f_{g}=f_{n}$ (nominal frequency case) which is a special case of the first case.

Int J Pow Elec \& Dri Syst, Vol. 10, No. 2, June 2019 : 1034 - 1048 


\subsection{The first case: $\boldsymbol{f}_{\boldsymbol{g}} \neq \boldsymbol{f}_{\boldsymbol{n}}$ (off-nominal frequency case)}

For $\left|f_{g}-f_{n}\right|<f_{n}$, and using suitable trigonometric identities it can be proven that $x_{i}$ in (4) (let us call it the direct component) and $y_{i}$ in (5) (let us call it the quadratic component) are functions of time and frequencies $f_{g}, f_{n}$ that are given as follows:

For phase a:

$$
\begin{aligned}
& x_{a}(t)=k_{1} \cos \left[\omega_{D} t+\theta_{0}+\Delta \theta_{1}\right]-k_{2} \cos \left[\omega_{T} t+\theta_{0}+\Delta \theta_{2}\right] \\
& y_{a}(t)=k_{1} \sin \left[\omega_{D} t+\theta_{0}+\Delta \theta_{1}\right]+k_{2} \sin \left[\omega_{T} t+\theta_{0}+\Delta \theta_{2}\right]
\end{aligned}
$$

For phase $b$ :

$$
\begin{aligned}
& x_{b}(t)=k_{1} \cos \left[\omega_{D} t+\theta_{0}+\Delta \theta_{1}\right]-k_{2} \cos \left[\omega_{T} t+\theta_{0}+\Delta \theta_{2}+2 p i / 3\right] \\
& y_{b}(t)=k_{1} \sin \left[\omega_{D} t+\theta_{0}+\Delta \theta_{1}\right]+k_{2} \sin \left[\omega_{T} t+\theta_{0}+\Delta \theta_{2}+2 p i / 3\right]
\end{aligned}
$$

And for phase c:

$$
\begin{aligned}
& x_{c}(t)=k_{1} \cos \left[\omega_{D} t+\theta_{0}+\Delta \theta_{1}\right]-k_{2} \cos \left[\omega_{T} t+\theta_{0}+\Delta \theta_{2}-2 p i / 3\right] \\
& y_{c}(t)=k_{1} \sin \left[\omega_{D} t+\theta_{0}+\Delta \theta_{1}\right]+k_{2} \sin \left[\omega_{T} t+\theta_{0}+\Delta \theta_{2}-2 p i / 3\right]
\end{aligned}
$$

where

$$
\begin{aligned}
& \omega_{D}=2 \pi\left(f_{g}-f_{n}\right) \\
& \omega_{T}=2 \pi\left(f_{g}+f_{n}\right) \\
& \Delta \theta_{1}=\frac{\omega_{D}}{2 f_{n}} \\
& k_{1}=\frac{\mathrm{E} \sin \left(\Delta \theta_{1}\right)}{2 \Delta \theta_{1}} \\
& \Delta \theta_{2}=\frac{\omega_{T}}{2 f_{n}} \\
& k_{2}=\frac{\mathrm{E} \sin \left(\Delta \theta_{2}\right)}{2 \Delta \theta_{2}}
\end{aligned}
$$
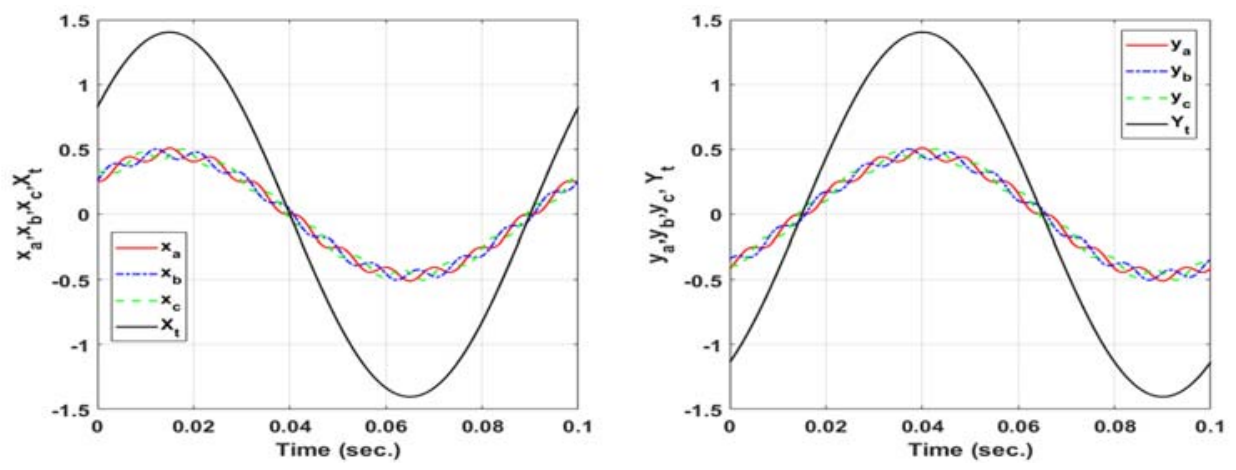

Figure 1. Waveforms of $x_{i}(t), x_{t}(t), y_{i}(t), y_{t}(t): E=1 \mathrm{~V}, f_{g}=60 \mathrm{~Hz}, f_{n}=50 \mathrm{~Hz}$ and $\theta_{0}=-\pi / 2$ 
It can be noticed that both of the direct and quadratic component $x_{i}$ and $y_{i}$ for the three-phases $(i=$ $\mathrm{a}, \mathrm{b}, \mathrm{c}$ ) as shown in (6) to (11) are composed of two sinusoidal terms. One of them is larger in magnitude and its frequency $\left(\omega_{D}\right)$ is the difference between the grid actual and nominal (or filter) frequency (if the two frequencies become equal, then it is a DC value as will be shown in (6), (7) of the second case). The other has a much smaller magnitude and its frequency $\left(\omega_{T}\right)$ is the sum of the grid actual and nominal frequency (if the two frequencies become equal, then it vanishes as the magnitude becomes zero as will be confirmed in the second case). Referring to the second term in the right hand side of each of (6), (8) and (10), it is evident that the three terms constitute a balanced three-phase set in cosine notation whose sum is zero. Thanks to the proper selection of the sine and cosine filter phase angles. Referring to the second term in the right hand side of each of (7), (9) and (11), the three terms also constitute a balanced three-phase set in sine notation whose sum is zero. Here it becomes clear the usefulness of the summing process combined with the proper selection of the FT filter phase angles. The summing process thus results in filtering the double-frequency oscillations without any additional or post-filtering. Some representative values $\left(E=1, f_{g}=60 \mathrm{~Hz}, f_{n}=50 \mathrm{~Hz}\right.$ and $\left.\theta_{0}=-\pi / 2\right)$ are used to plot

Figure 1 where the waveforms of $x_{i}$ and $y_{i}$ are plotted in the left subplot and the right subplot respectively against time to illustrate the double-frequency ripple, the sums $x_{t}$ and $y_{t}$ are also plotted in the two subplots to show the smooth sinusoidal variations as the three higher frequency oscillations running at $\left(f_{g}+f_{n}\right)$ cancel each other. Therefore, the sum of the direct $x_{t}$ and the quadratic components $y_{t}$ for the three individual phases, are given by (18) and (19), respectively:

$$
\begin{aligned}
& \mathrm{x}_{\mathrm{t}}=\sum_{\mathrm{i}=\mathrm{a}}^{\mathrm{c}} \mathrm{x}_{\mathrm{i}}=3 \mathrm{k}_{1} \cos \left[\omega_{\mathrm{D}} \mathrm{t}+\theta_{0}+\Delta \theta_{1}\right] \\
& \mathrm{y}_{\mathrm{t}}=\sum_{\mathrm{i}=\mathrm{a}}^{\mathrm{c}} \mathrm{y}_{\mathrm{i}}=3 \mathrm{k}_{1} \sin \left[\omega_{\mathrm{D}} \mathrm{t}+\theta_{0}+\Delta \theta_{1}\right]
\end{aligned}
$$

Dividing (19) by (18) yields:

$$
\frac{\mathrm{y}_{\mathrm{t}}}{\mathrm{x}_{\mathrm{t}}}=\tan \left[\omega_{\mathrm{D}} \mathrm{t}+\theta_{0}+\Delta \theta_{1}\right]
$$

Therefore:

$$
2 \pi\left(f_{g}-f_{n}\right) t+\theta_{0}+\Delta \theta_{1}=\tan ^{-1}\left(\frac{y_{t}}{x_{t}}\right)
$$

The phase angle $\theta_{a}$ of the grid voltage $V_{g a}$ that is equal to $2 \pi f_{g} t+\theta_{0}$ from (1) is then given by rearranging (21) as follows:

$$
\theta_{\mathrm{a}}=2 \pi \mathrm{f}_{\mathrm{g}} \mathrm{t}+\theta_{0}=\tan ^{-1}\left(\frac{\mathrm{y}_{\mathrm{t}}}{\mathrm{x}_{\mathrm{t}}}\right)+2 \pi \mathrm{f}_{\mathrm{n}} \mathrm{t}-\Delta \theta_{1}
$$

Substituting from (14) into (22) yields,

$$
\theta_{a}=\tan ^{-1}\left(\frac{y_{t}}{x_{t}}\right)+2 \pi f_{n} t-\frac{\pi\left(f_{g}-f_{n}\right)}{f_{n}}
$$

Recalling that $f_{g}$ is constant, then $\frac{\Delta \mathrm{f}_{\mathrm{g}}}{\Delta \mathrm{t}}=0$ and $\Delta \theta_{1}$ is constant. $\theta_{0}$ is also constant. Therefore, the time derivatives of both $\Delta \theta_{1}$ and $\theta_{0}$ are typically zeros. Consequently, the value of $f_{g}$ can be calculated by taking time derivative of both sides of (22) as follows:

$$
f_{g}=\frac{1}{2 \pi} \cdot \frac{d}{d t}\left(\tan ^{-1}\left(\frac{y_{t}}{x_{t}}\right)\right)+f_{n}
$$

Once $f_{g}$ is estimated, $\Delta \theta_{1}$ is determined and consequently $\theta_{a}$ can be estimated from (22). To estimate the magnitude $E$, it can be found from (15), (18) and (19) that:

Int J Pow Elec \& Dri Syst, Vol. 10, No. 2, June 2019 : 1034 - 1048 


$$
\mathrm{E}=\frac{2}{3} \cdot \frac{\Delta \theta_{1}}{\sin \left(\Delta \theta_{1}\right)} \cdot \sqrt[2]{\mathrm{x}_{\mathrm{t}}^{2}+\mathrm{y}_{\mathrm{t}}^{2}}
$$

The term $\frac{\Delta \theta_{1}}{\sin \left(\Delta \theta_{1}\right)}$ can be called the magnitude correction factor and the term $\frac{2}{3} \sqrt[2]{x_{t}^{2}+y_{t}^{2}}$ can be called the uncompensated magnitude.

\subsection{Second case: $\boldsymbol{f}_{\boldsymbol{g}}=\boldsymbol{f}_{\boldsymbol{n}}$ (nominal frequency case)}

By using suitable trigonometric identities, it can be proven that $x_{i}$ and $\mathrm{y}_{\mathrm{i}}$ are independent of time and are given as follows:

$$
\begin{aligned}
& x_{a}=x_{b}=x_{c}=\frac{E \cos \left(\theta_{0}\right)}{2} \\
& y_{a}=y_{b}=y_{c}=\frac{E \sin \left(\theta_{0}\right)}{2}
\end{aligned}
$$

Therefore, the summing for $x$ and $y$ components of the three-phase are given by (28) and (29), respectively:

$$
\begin{aligned}
& x_{t}=\sum_{i=a}^{c} x_{i}=\frac{3 \mathrm{E} \cos \left(\theta_{0}\right)}{2} \\
& y_{t}=\sum_{i=a}^{c} y_{i}=\frac{3 \mathrm{E} \sin \left(\theta_{0}\right)}{2}
\end{aligned}
$$

Now dividing both sides in (29) by their respective sides in (28) yields:

$$
\begin{aligned}
& \frac{y_{t}}{x_{t}}=\frac{\sin \left(\theta_{0}\right)}{\cos \left(\theta_{0}\right)}=\tan \left(\theta_{0}\right) \\
& \theta_{0}=\tan ^{-1}\left(\frac{y_{t}}{x_{t}}\right)
\end{aligned}
$$

Recalling the equality of $f_{n}$ and $f_{g}$, the phase angle $\theta_{a}$ of grid voltage $V_{g a}$ is then given by (32):

$$
\theta_{a}=2 \pi f_{g} t+\theta_{0}=2 \pi f_{n} t+\tan ^{-1}\left(\frac{y_{t}}{x_{t}}\right)
$$

According to (23), the last term in the right-hand side of (23) vanishes when $f_{g}=f_{n}$ thus leading to (32) and confirms that the nominal frequency case is a special case of the off-nominal frequency case. Therefore, it is obvious that (23) can be generalized for both nominal and off-nominal frequency cases. Thus, phase angle tracking is a seamless process when the actual frequency shifts from nominal to off-nominal mode and vice versa during actual operation.

The magnitude $E$ can simply obtained from (28) and (29) as follows:

$$
E=\frac{2}{3} \cdot \sqrt[2]{x_{t}^{2}+y_{t}^{2}}
$$

Knowing that: $\lim _{\Delta \theta_{1} \rightarrow 0} \frac{\Delta \theta_{1}}{\sin \left(\Delta \theta_{1}\right)}=1$, the (25) becomes the same as (33) and it turns as stated earlier that the nominal frequency case is a special case of the off-nominal generic case. Therefore (25) can be a valid expression for both cases. Thus, the magnitude tracking is also a seamless process should the actual grid frequency shifts from off-nominal to nominal mode of operation and vice versa.

Now it is clear that by proper selection of the phase sequence and phase shift of the waveforms used for sine and cosine filters of the FT and applying the summing procedure we get a very effective filtering for the double-frequency oscillation characterizing the FT in off-nominal frequency case. Figure 2 illustrates the schematic diagram of the proposed FT implementation based on (1) through (33). In the leftmost block, the

Frequency adaptive sliding fourier transform for synchronizing VSI to the grid (Osama M. Arafa) 
sine and cosine filters are applied to the three-phase voltage, and the summing procedure is applied to get $x_{t}$ and $y_{t}$. The uncompensated phase angle given by (21) is estimated in the leftmost block as well. The $f_{g}$ is estimated according to (24) in the intermediate block. Using the estimated $f_{g}$, the phase angle correction term $\Delta \theta_{1}$ can be estimated in the rightmost block. Subtracting the correction term from the uncompensated phase angle as per (23) results in the true phase angle of the grid voltage $V_{g a}$. The magnitude correction factor can be easily calculated also from (25) in the bottom block and finally the compensated magnitude can be estimated.

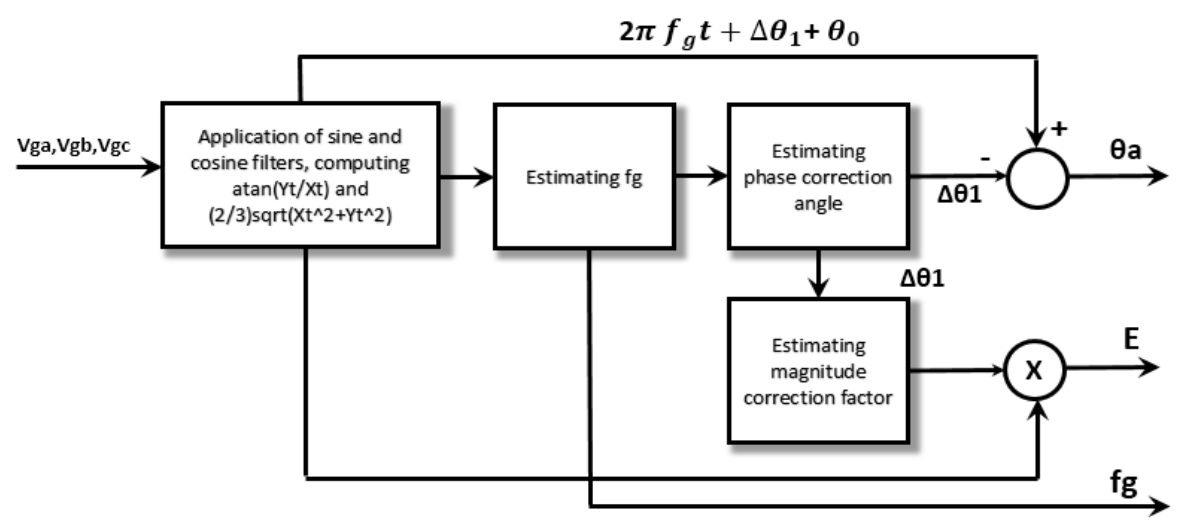

Figure 2. Schematic diagram of the proposed FT implementation for three-phase system

\section{SLIDING FT IMPLEMENTATION (SFT)}

The sliding integrations from $t$ to $t+T_{n}$ as mentioned in (4) and (5) can be carried out numerically in a discrete sampled system. The method applied for obtaining and updating the numerical integration over one cycle upon picking each fresh sample of $x i$ (or $y i$ ) is to insert a delaying buffer of length $T_{n}$ after the discrete time integrator [15]. Thus, integration over $T_{n}$ is updated every new sample of $x i$ and it is implemented as shown schematically in Figure. In this implementation, the buffer is initialized with zeroes and it takes $T_{n} / T_{s}$ samples to be filled incrementally with valid readings as $T_{s}$ is equal to the sampling period. Based on this, the detected grid angle and frequency needs one complete cycle $\left(T_{n}\right)$ to converge to their actual values for all $f_{g} \neq f_{n}$. It can be shown that for $f_{g}=f_{n}$ the detection is almost instantaneous.

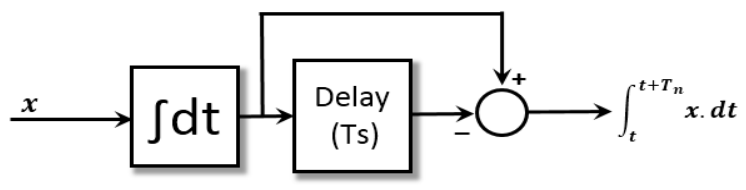

Figure 3. Updating Numerical integration over one $T_{n}$ cycle upon each new sample of $x$

\section{THE PROPOSED SFT-PLL}

The implementation of SFT shown in Figure 2 works very fine and gives fast and accurate results in the nominal and off-nominal frequency cases as long as the input voltages are free from harmonic distortion Once the input frequency shifts from nominal value while harmonics are existing the estimated phase angle, the estimated magnitude and the estimated frequency all suffer from oscillations due to spectral leakage and the SFT loses its excellent harmonic rejection and becomes unpractical. This is shown using numerical simulation in Figure 4. Figure 4 (a) illustrates that the input voltage is distorted with harmonics starting from $t=0.1 \mathrm{sec}$ to $t=0.45 \mathrm{sec}$ and the frequency is stepped up then down by $10 \mathrm{~Hz}$ at $t=0.15$ and $t=0.35 \mathrm{sec}$ respectively. Figure 4 (b) and (c) show that the SFT rejects the harmonics after exacty one power cycle and gives accurate frequency and phase angle estimation when it works at nominal frequency $(50 \mathrm{~Hz})$ i.e. from

Int J Pow Elec \& Dri Syst, Vol. 10, No. 2, June 2019 : 1034 - 1048 
$t=0.05 \mathrm{sec}$ to $t=0.15 \mathrm{sec}$ and from $t=0.35 \mathrm{sec}$ to $t=0.5 \mathrm{sec}$. However, during the off-nominal period from $t=0.15 \mathrm{sec}$ to $t=0.35 \mathrm{sec}$, the existence of harmonics characterizes the estimation of both frequency and phase angle by a sustained error.

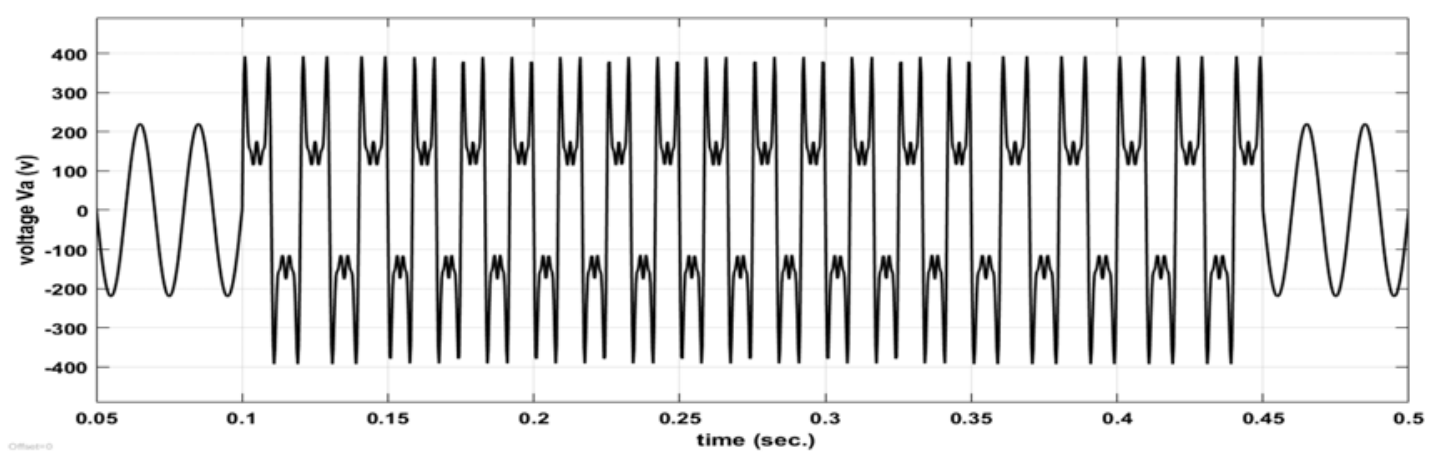

(a)

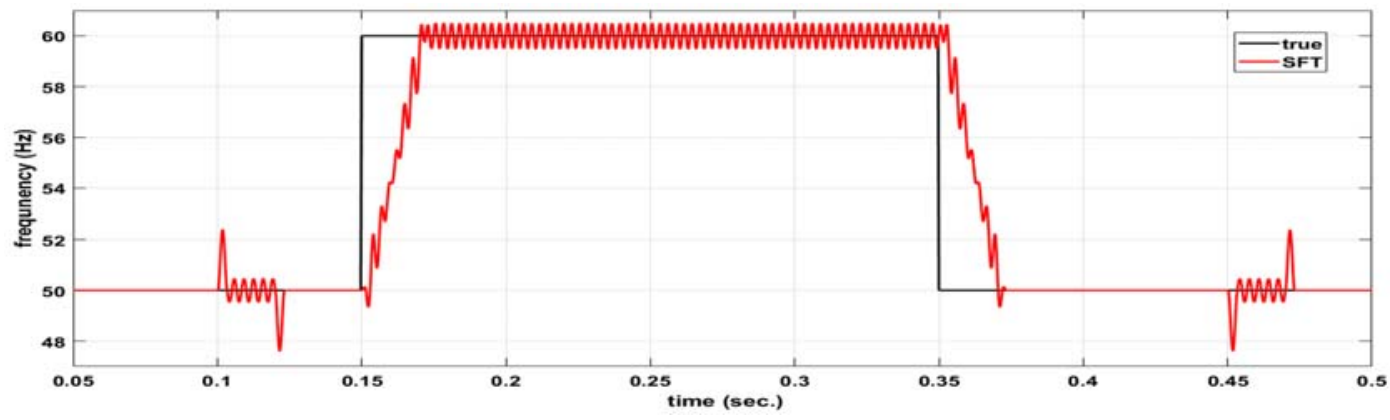

(b)

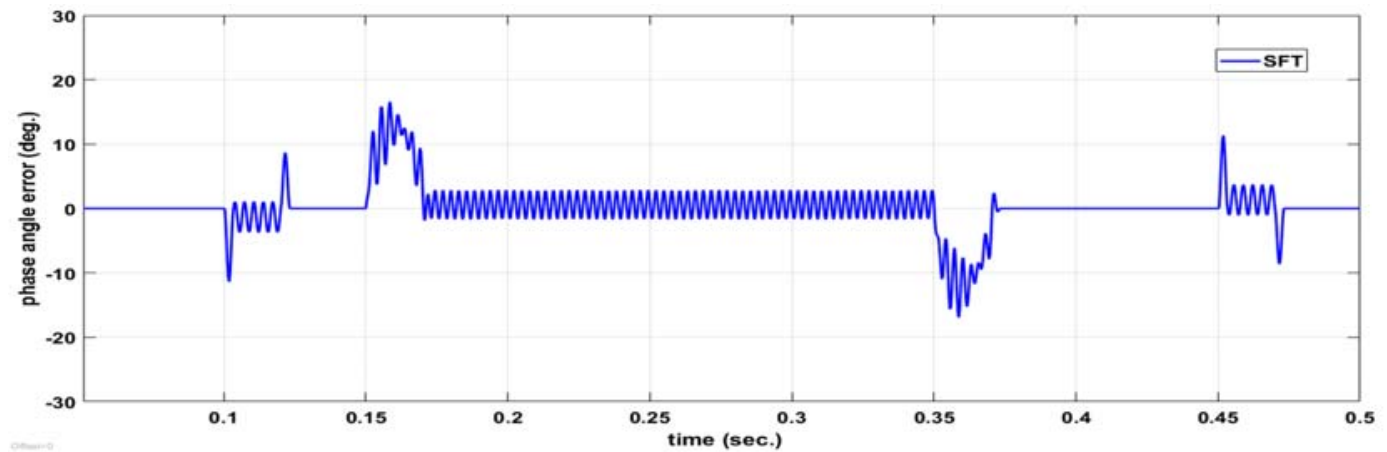

(c)

Figure 4. (a) Voltage of phase a (other phases omitted for clarity), (b) estimated frequency by SFT (c) error in phase angle estimation by SFT

To overcome this deficit, and considering the ability of the FT to detect the phase difference $\theta_{0}$ between the angle of the sine/cosine filters and the angle of the input voltage as shown by (23), the SFT can be used as a phase detector in a standard phase locked loop. To do this, the SFT time window (or number of processed samples) should be continuously and properly adjusted to enforce nominal-frequency operation a $\mathrm{n}$ and hence maintain its excellent harmonic rejection. This is schematically shown in Figure 5 and Figure 6. Note that the shaded rectangles in phase $\mathrm{b}$ and $\mathrm{c}$ in Figure 5 are similar in construction to that of phase a. The phase-locked loop PID controller in Figure 6 sets the loop frequency so as to eliminate the phase difference $\Delta \theta$. The frequency $\widehat{f}_{g}$ and the phase angle $\widehat{\theta_{g}}$ estimated by the PLL are fed back to the variable window width

Frequency adaptive sliding fourier transform for synchronizing VSI to the grid (Osama M. Arafa) 
SFT to calculate the sine and cosine filters whose outputs are used to explore the phase difference $\Delta \theta$. The PLL therefore adjusts the frequency of the SFT such that when it locks, the SFT works at the actual grid frequency. In this case, the filter frequency $f_{n}$ shifts to $f_{g}$ and the operation mode shifts from off-nominal to nominal and $\Delta \theta$ vanishes. This arrangement provides fast phase difference detection while maintaining the SFT well-known excellent harmonic rejection. Figure 5 and Figure 6 together illustrate the structure of the proposed SFT-PLL technique.

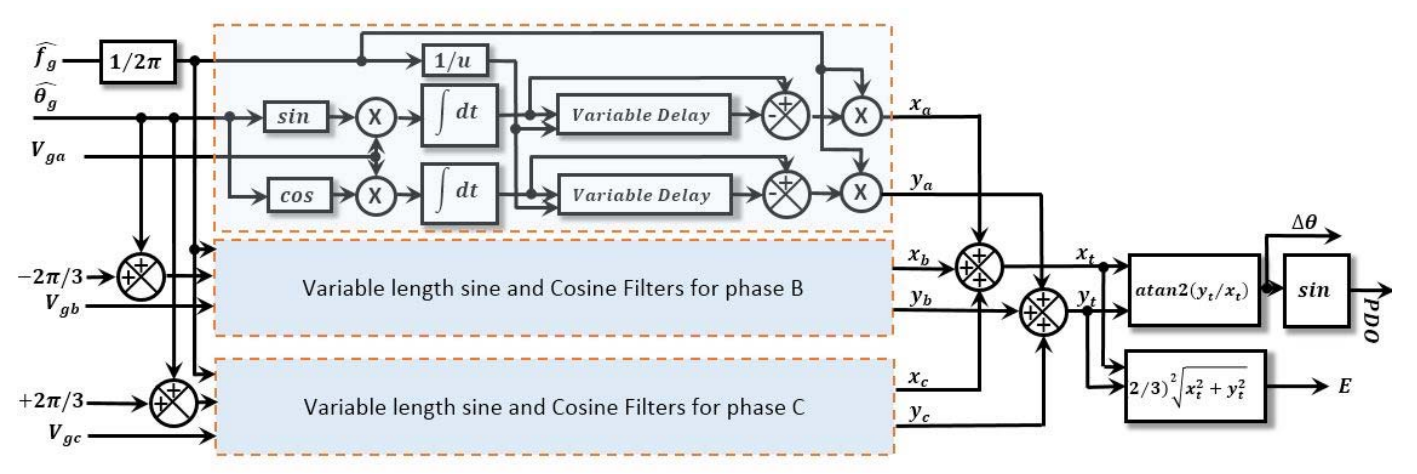

Figure 5. Schematic representation of the proposed variable window width SFT phase detector

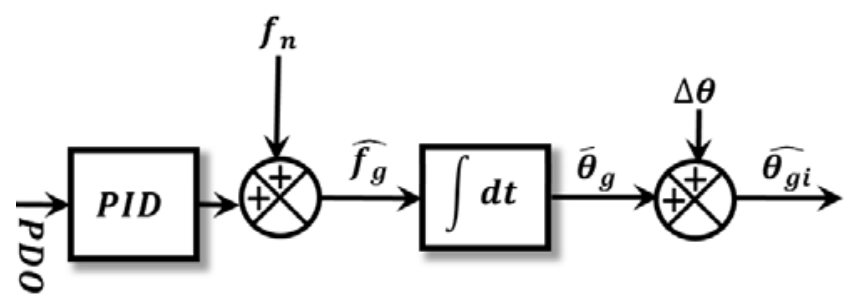

Figure 6. Phase-locked loop schematic representation

\section{EXPERIMENTAL TESTING METHODOLOGY \& RESULTS}

The experimental testing rnsures the applicability of the proposed technique in real-time setting and under practical operating conditions that may violate some of the considered assumptions made during the mathematical analysis. The testing method depends on generating the voltage vectors that contains the target test characteristics (DC offset, harmonic distortion, magnitude, frequency or phase jump and unbalance of the three-phases) using offline MATLAB simulation. These voltage vectors are sampled at 64 sample per power cycle $(0.02 \mathrm{sec})$ and recorded along with an array of corresponding timestamps. Also the true phase angle, the true frequency and the true magnitude which areused for generating them are sampled and recorded for sake of comparison and validation. Then these voltage vectors are played back in real-time at the same sampling rate using dSPACE ds1202 DSP D/A channels, read back via A/D channels and are used as inputs to the SFT-PLL. The output of the SFT-PLL are then recorded in MAT files, then compared with the true values that are pre-recorded to compute the errors. The same procedure is repeated with $\alpha \beta \beta P L L$ technique and the error in both cases are plotted on same axis to compare the performance of both techniques. The testing voltages are:

a. $\quad 50 \mathrm{~Hz}$ balanced three-phase (peak $E=220 \mathrm{~V}$ ) with the following testing scenarios: Harmonic distortion (composed of third, fifth, seventh and ninth harmonic components with $70 \%, 60 \%, 30 \%$ and $20 \%$ of the fundamental magnitude respectively) switched-on at $t=1 \mathrm{sec}$ and switched- off at $t=1.5 \mathrm{sec}$. Phase jump of $\pi / 2$ at $t=2 \mathrm{sec}$, frequency step-up of $5 \mathrm{~Hz}$ at $t=2.5 \mathrm{sec}$. Magnitude step- up of $0.2 E$ at $t=3.5 \mathrm{sec}$. Adding DC offsets to balanced three-phase at $t=4 \mathrm{sec}, 0.1 \mathrm{E}$ to phase $\mathrm{A},-.05 \mathrm{E}$ to phase $\mathrm{B},-0.05 \mathrm{E}$ to phase C.

Int J Pow Elec \& Dri Syst, Vol. 10, No. 2, June 2019 : 1034 - 1048 
b. Unbalanced three-phase input with knowing symmetrical components structure as follows: magnitude of positive sequence is $220 \mathrm{~V}$ and its angle is zero, magnitude of negative sequence is $60 \mathrm{~V}$ and its angle is -150 deg., magnitude of zero sequence is $20 \mathrm{~V}$ and its angle is $10 \mathrm{deg}$.

In all of these tests, the error is computed as the difference between the true quantity and the estimated one. In the case of unbalanced three-phase, the true value for phase angle estimation error is the phase angle of phase $\mathrm{A}$ of the positive sequence.

Harmonic distortion test: Figure 7 (a) shows the introduction of harmonics at $t=1 \mathrm{sec}$. Figure 7 (b) and (c) show that $\alpha \alpha \beta$ PLL gives continuous oscillation in estimation of both frequency $( \pm 20 \mathrm{~Hz})$ and angle ( \pm 4 deg.) while SFT-PLL gives oscillation in estimated frequency $( \pm 1 \mathrm{~Hz})$ for only one power cycle. Oscillation in estimated angle continues for one power cycle and error settles to zero in four cycles.

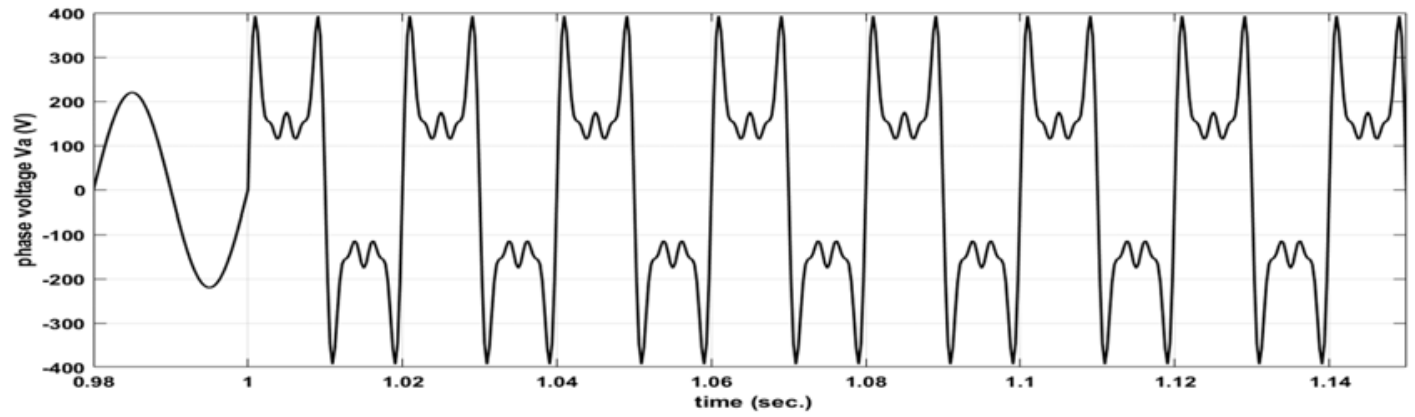

(a)

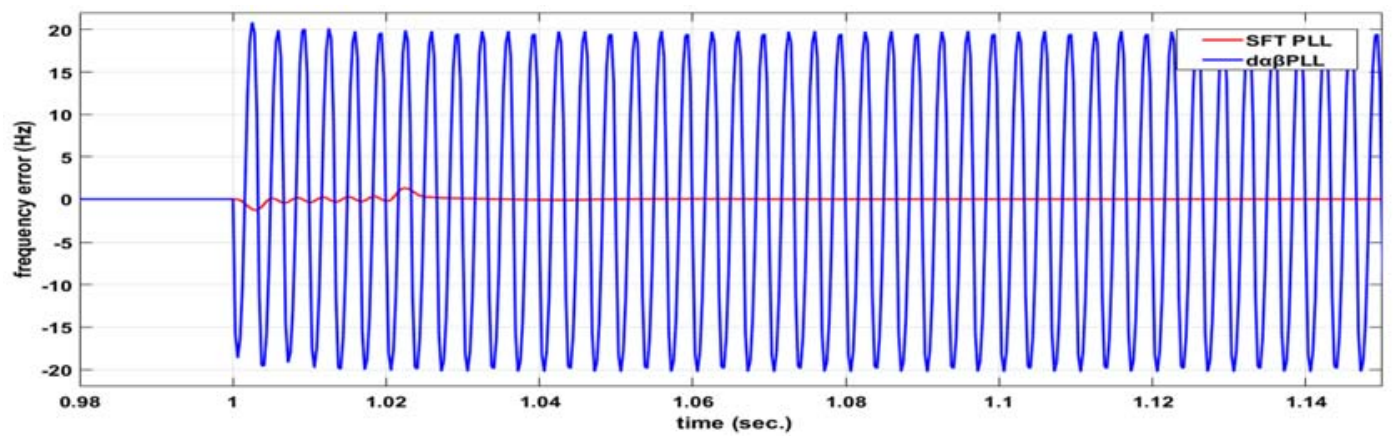

(b)

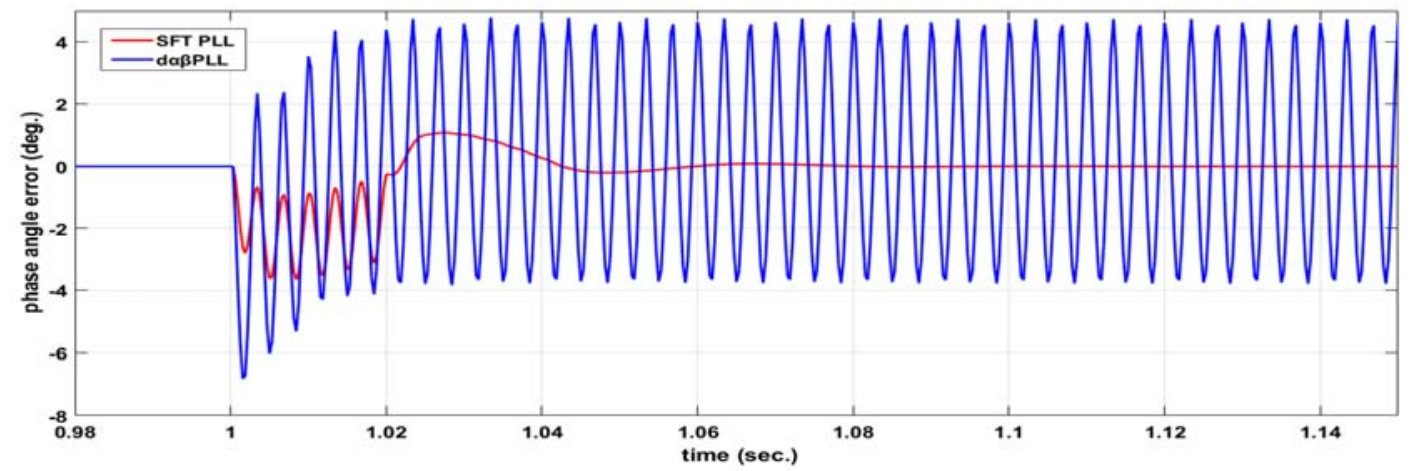

(c)

Figure 7 (a) Voltage of phase A, harmonics switched on at $t=1 \mathrm{sec}$, (b) frequency estimation error, harmonics switched on at $t=1 \mathrm{sec}$, (c) phase angle estimation error, harmonics switched on at $t=1 \mathrm{sec}$ 
Phase jump test: In this test a $\pi / 2$ phase jump at $t=2 \mathrm{sec}$ is done. Figure 8 (a) and (b) show that $\mathrm{d} \alpha \beta$ PLL frequency error peak is $28.6 \mathrm{~Hz}$. The frequency error settles to zero in more than 6 power cycles. Phase angle error settles to zero in more than 6 power cycles. SFT-PLL results in frequency error peak of 13.5 Hz. The frequency error settles to zero in less than 5 power cycles. Phase angle error settles to zero within 5 power cycles. Hence, SFT-PLL gives superior performance.

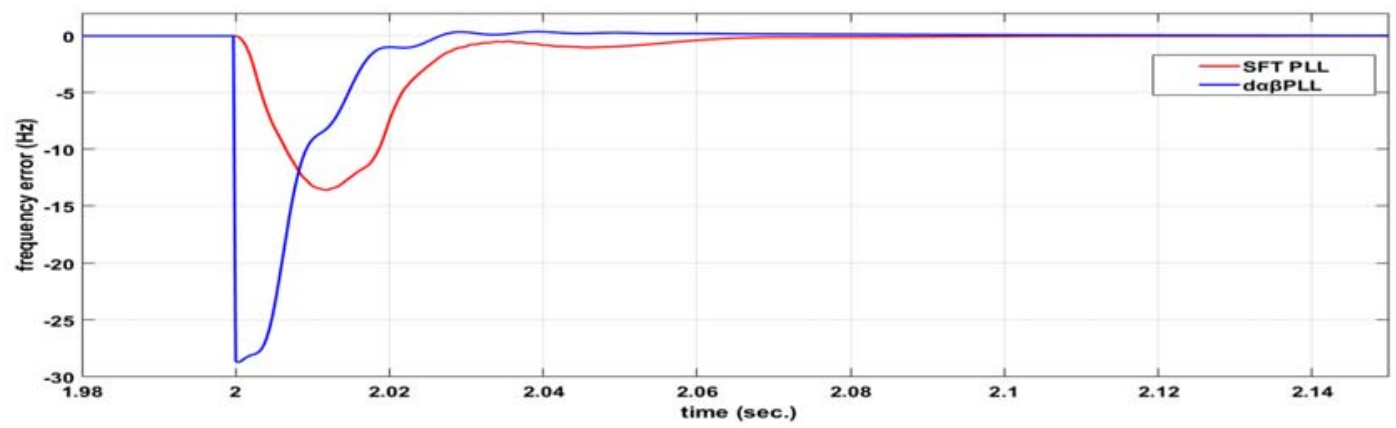

(a)

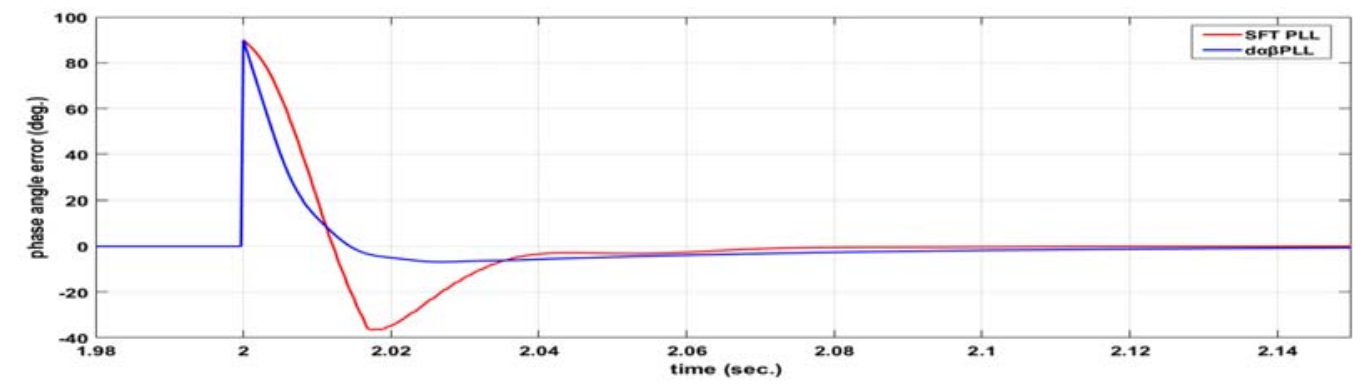

(b)

Figure 8 (a) frequency estimation error, $\pi / 2$ phase jump at $t=2 \mathrm{sec}$, (b) phase angle estimation error, $\pi / 2$ phase jump at $t=2 \mathrm{sec}$

Frequency step change test: Figure 9 (a), and (b) show that for $d \alpha \beta P L L$, the frequency estimation error settles to zero in more than 8 power cycles. Peak phase angle error is $8.2 \mathrm{deg}$. The error in phase angle settles to zero in more than 10 power cycles. For SFT-PLL, the frequency estimation error settles to zero in 5 power cycles. Peak phase angle error is $12.8 \mathrm{deg}$. Error settles to zero in 5 power cycles. Therefore, SFT-PLL errors settle faster than those of $d \alpha \beta P L L$.

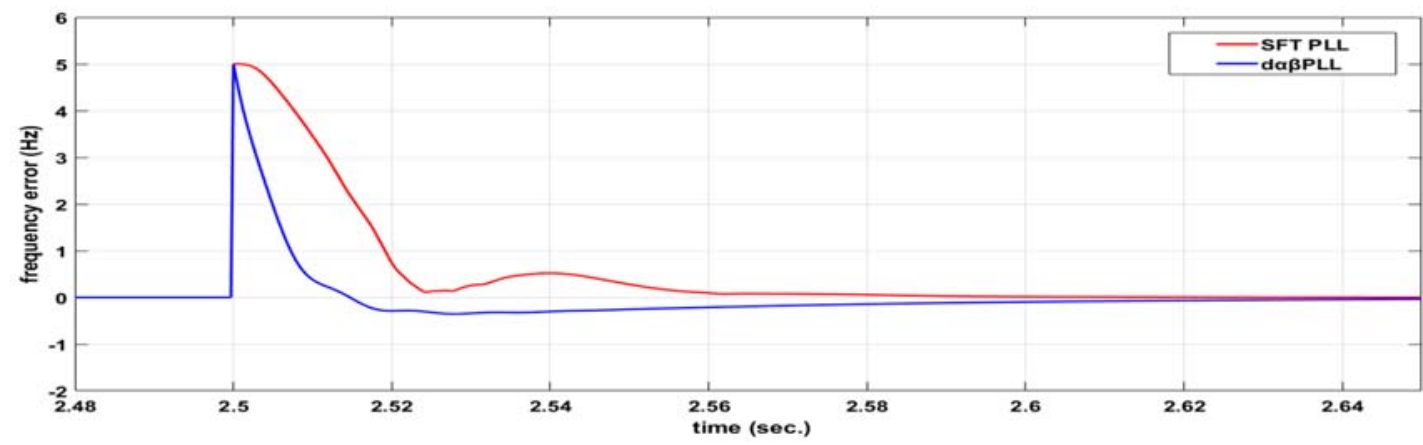

(a) 
Figure 9 (a). Frequency estimation error, $5 \mathrm{~Hz}$ frequency step up at $t=2.5 \mathrm{sec}$

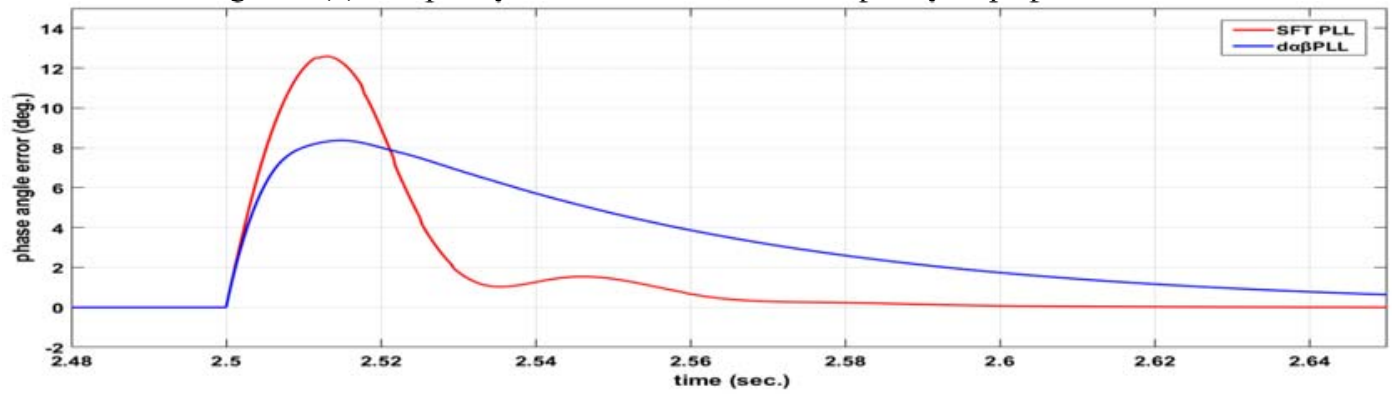

(b)

Figure 9. (a) frequency estimation error, $5 \mathrm{~Hz}$ frequency step up at $t=2.5 \mathrm{sec}$, (b) phase angle estimation error, $5 \mathrm{~Hz}$ frequency step up at $t=2.5 \mathrm{sec}$

Magnitude step change test: In this test, a $20 \%$ step change in magnitude is done at $t=3.5 \mathrm{sec}$. Figure 10 (a) and (b) show that d $\alpha \beta$ PLL results in decayed oscillation in frequency with a peak $-0.75 \mathrm{~Hz}$ and lasting for 4 power cycles. Phase angle error of $1 \mathrm{deg}$. decays to zero in 4 power cycles. While for SFT-PLL there is no error at all in frequency or phase angle. SFT-PLL is thus more robust against magnitude disturbances than $\alpha \alpha \beta$ PLL.

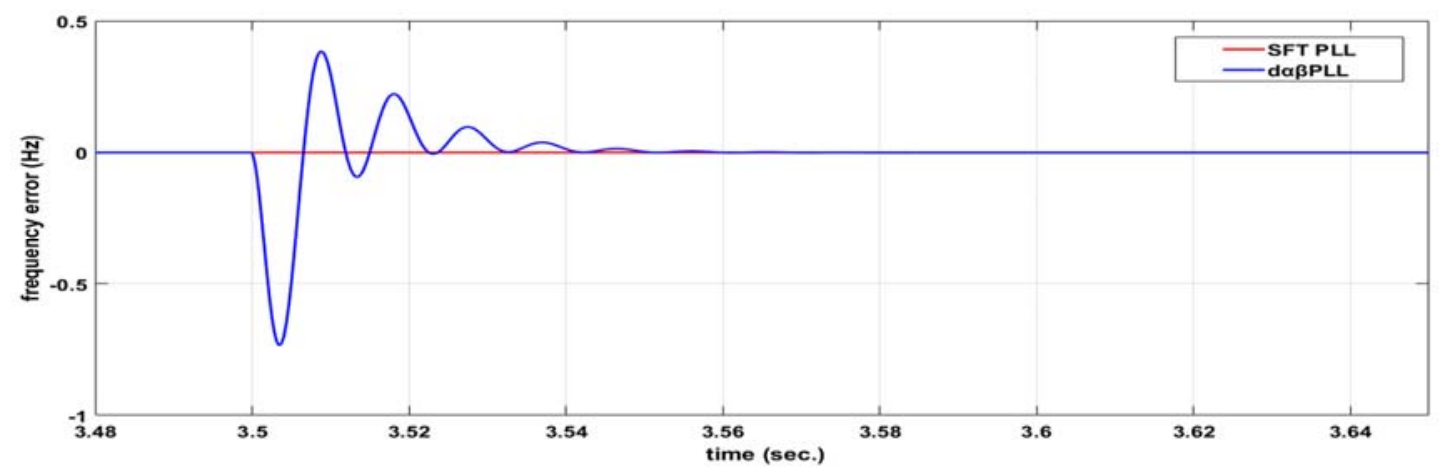

(a)

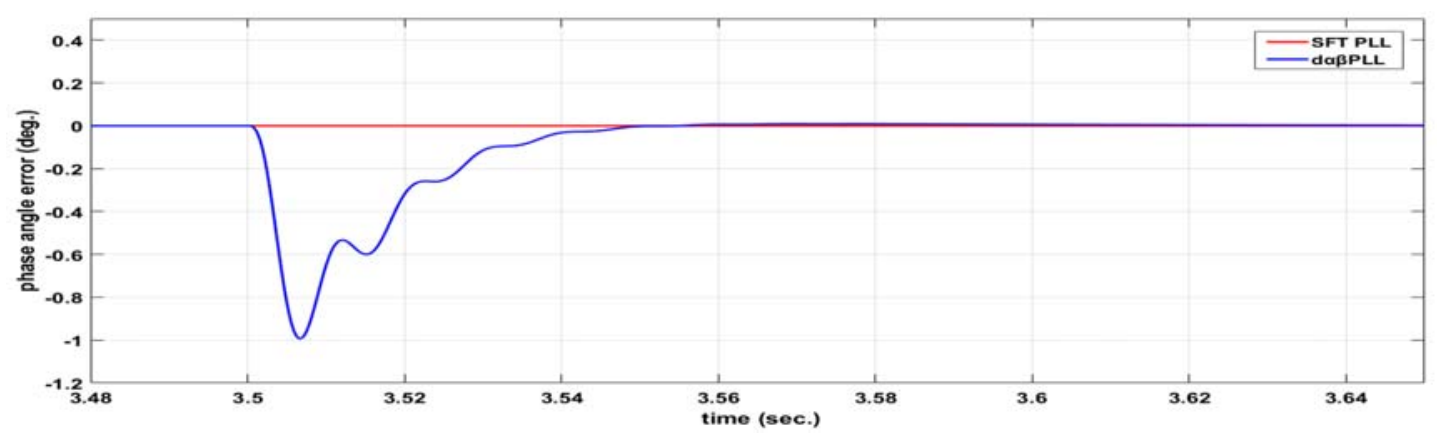

(b)

Figure 10. (a) Frequency estimation error, 20\% step up in magnitude at $t=3.5 \mathrm{sec}$, (b) phase angle estimation error, $20 \%$ step up in magnitude at $t=3.5 \mathrm{sec}$

DC-offset test: In this test, the three phase voltages are affected with the prescribed DC offsets at $t=4 \mathrm{sec}$. Figure 11 (a) and (b) show that $d \alpha \beta$ PLL results in continuous oscillation in frequency error of \pm 2.2 
$\mathrm{Hz}$ and in phase angle error of $\pm 2 \mathrm{deg}$. While for SFT-PLL the oscillation in frequency error and phase angle error has a smaller magnitude and noticeable only within two and four power cycles respectively. SFT-PLL is thus more robust against DC offset disturbance than $d \alpha \beta$ PLL.

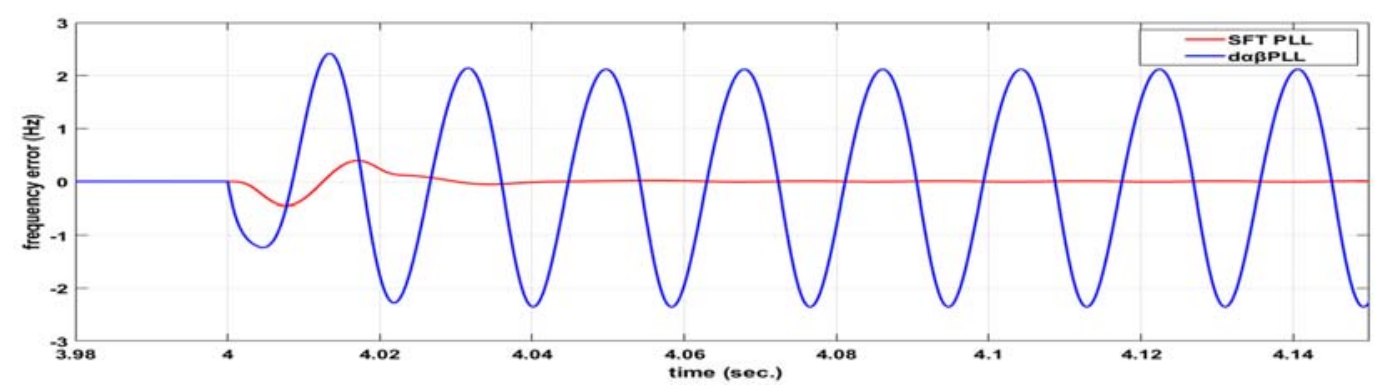

(a)

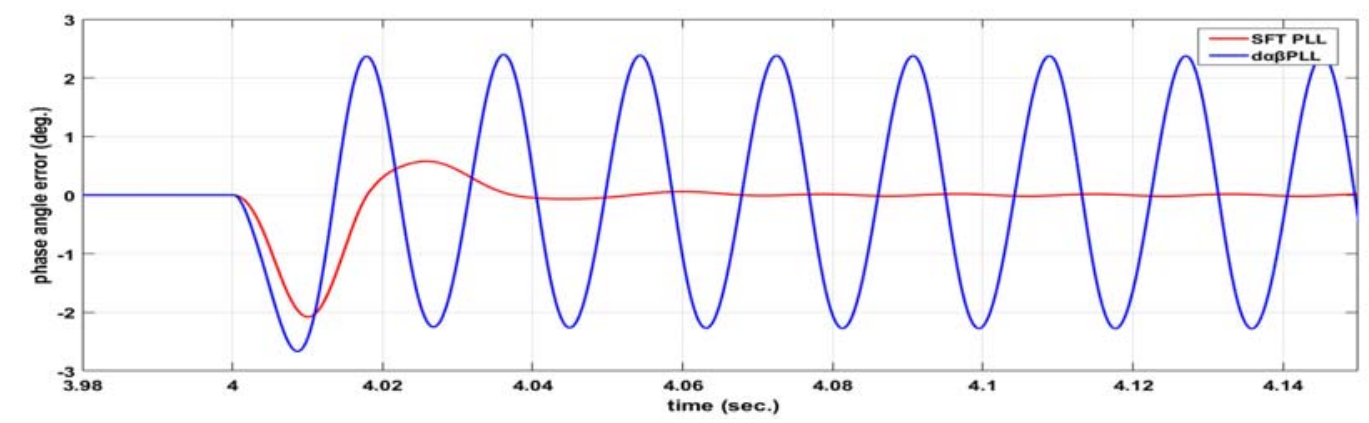

(b)

Figure 11. (a) Frequency estimation error, three-phase voltage with DC offset (Va+20, Vb-10, Vc-10), (b) phase angle estimation error, three-phase voltage with DC offset $(\mathrm{Va}+20, \mathrm{Vb}-10, \mathrm{Vc}-10)$

Unbalanced three-phase test: Figure 12 (a) shows the three-phase voltages with the prescribed unbalance. Figure12 (b) and (c) show that $d \alpha \beta$ PLL results in continuous oscillation in frequency error $(-2.9$ $\mathrm{Hz}:+2.6 \mathrm{~Hz}$ ) and in phase angle error of $\pm 3 \mathrm{deg}$. For SFT-PLL, the decays to zero in 4 power cycles. While for SFT-PLL there no errors at all in estimating frequency and phase angle. SFT-PLL is thus is completely immune against unbalance disturbance while $d \alpha \beta P L L$ is not. Note that the reference phase angle in this case is the positive sequence phase angle.

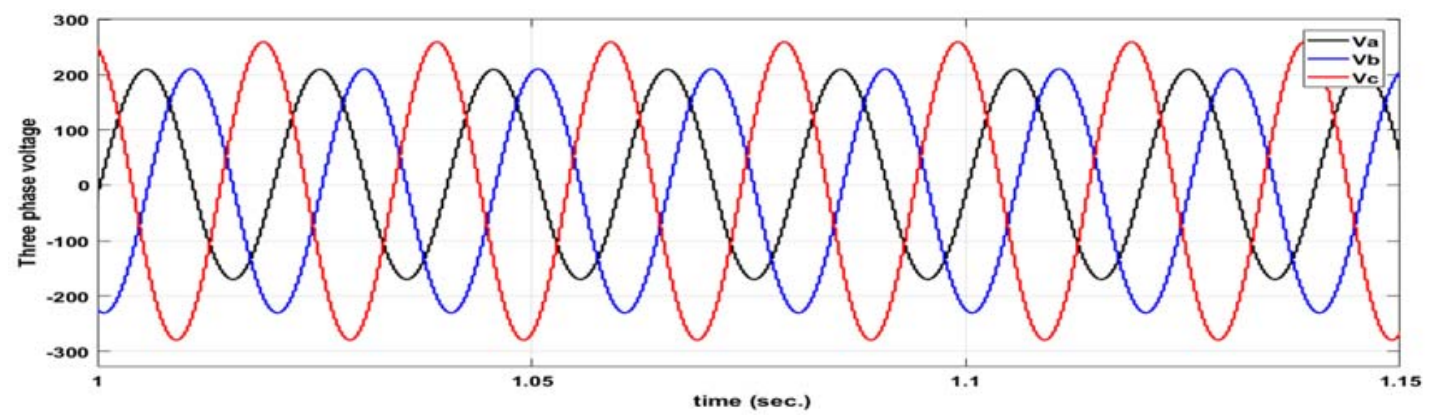

(a) 


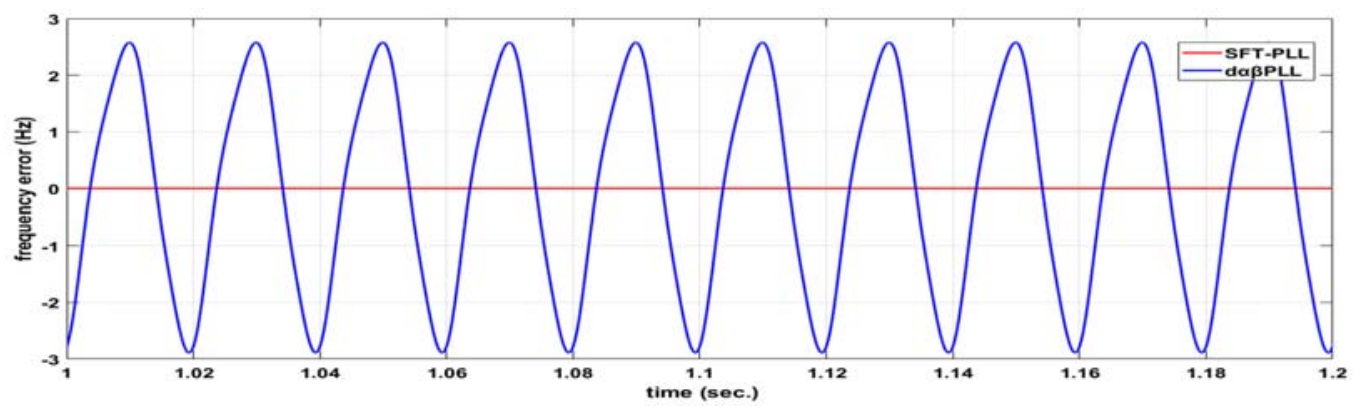

(b)

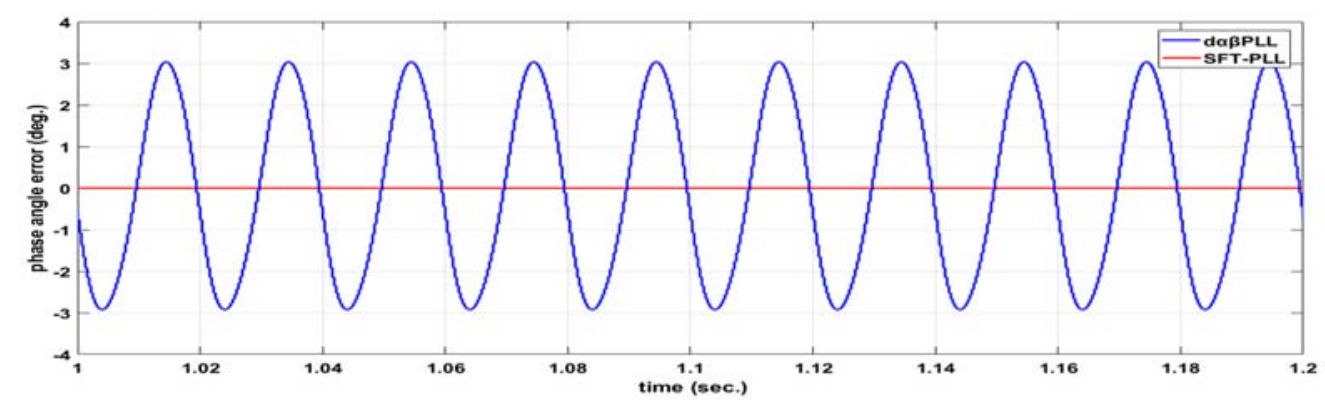

(c)

Figure 12. (a) three-phase unbalanced set, (b) frequency error in case of three-phase unbalanced input (c) frequency error in case of three-phase unbalanced input

\section{CONCLUSION}

The mathematical analysis in time domain of the SFT of a single frequency three-phase set shows that by properly setting the phase angle used in the sine/cosine filters of the individual phases and then by summing the direct components and the quadratic components independently, the double-frequency oscillation is completely removed without post filters. The phase angle error and the magnitude error are also analyzed and their values are determined analytically. Therefore, any errors in the phase angle and magnitude can be corrected. However, due to spectral leakage, the SFT shows excellent harmonic rejection when working at nominal frequency while it shows inferior performance at off-nominal frequency mode of operation when the input has a high harmonic content. The benefits of the excellent harmonic rejection of SFT at nominal frequency mode of operation are preserved by timely adjusting the SFT frequency using a phase-locked loop. The proposed SFT-PLL has a fixed sampling rate which is consistent with the design of common digital controllers. The proposed SFT-PLL has been tested and validated using DSP in real time. A comparative study with one of the best performing PLL methods d $\alpha \beta$ PLL under different practical conditions has been presented. The comparison demonstrates that SFT-PLL can successfully handle unbalanced threephase input and computes the positive sequence phase angle while d $\alpha \beta P L L$ gives a sustained oscillatory error in this case. The SFT-PLL is very resistant to magnitude disturbances while d $\alpha \beta P L L$ is affected by such disturbances. Indeed, the SFT-PLL outperforms the d $\alpha \beta P L L$ in the presence of harmonic distortion or/and DC offset. In a very wide range of disturbances, the SFT-PLL error settles to zero faster than that of the d $\alpha \beta$ PLL. The excellent performance strongly suggests the SFT-PLL for synchronizing renewable energy sources with the utility grid under challenging grid conditions like fault-ride-through periods.

\section{REFERENCES}

[1] Jaalam N., et al. "A comprehensive review of synchronization methods for grid-connected converters of renewable energy source." Renewable and Sustainable Energy Reviews, vol 59, no 2, pp. 1471-1481, 2016.

[2] Carugati I., et al. "Variable Sampling Period Filter PLL for Distorted Three-Phase Systems." IEEE Transactions on Power Electronics.Vol. 27, no. 1, pp. 321-330, 2012.

[3] Cho C., et al. "Active Synchronizing Control of a Microgrid." IEEE Transactions on Power Electronics. 2011: 26(12): 3707-3719. 
[4] Jain B., et al. "Control strategies of grid interfaced wind energy conversion system: An overview." Renewable and Sustainable Energy Reviews. Vol 47, no. 5, pp. 983-996, 2015.

[5] Vainio O. et al. "Noise reduction in zero crossing detection by predictive digital filtering." IEEE Transactions on Industrial Electronics. Vol. 42, no. 1, pp. 58-62, 1995

[6] Jaalam N., et al., Bakar A. H. A., Chia Kwang Tan, Ahmed M.A. Haidar. "A comprehensive review of synchronization methods for grid-connected converters of renewable energy source." Renewable and Sustainable Energy Reviews. Vol. 59, no. 2, pp. 1471-1481, 2016.

[7] Se-Kyo Chung. "A phase tracking system for three-phase utility interface inverters." IEEE Trans. Power Electronics. Vol. 15, no. 3, pp. 431-438, 2000.

[8] Sumathi S., et al. "Solar PV and Wind Energy Conversion Systems an Introduction to Theory, Modeling with MATLAB/SIMULINK, and the Role of Soft Computing." New York: Springer, 2015, chap. 7.

[9] Ghartemani M. K. et al. "A nonlinear adaptive filter for online signal analysis in power systems applications". IEEE Trans. Power Delivery. vol 17 no. 2, pp. 617-622, 2002.

[10] Guo X., et al. "Phase locked loop and synchronization methods for grid interfaced converters: a review." Przegląd Elektrotechniczny electrical review. 2011: 4(1): 182.

[11] Hsich G.-C. et al. "Phase-locked loop techniques-A survey". IEEE Trans. Industrial Electronics. Vol. 43 no. 2 , pp. 609-615, 1996.

[12] McGrath B., et al. "Power Converter Line Synchronization Using a Discrete Fourier Transform (DFT) Based on a Variable Sample Rate.” IEEE Transactions on Power Electronics. vol 20 no 4, pp. 877-884, 2005.

[13] Begovic M. M., et al. Frequency tracking in power networks in the presence of harmonics. IEEE Trans. Power Delivery.vol. 8, no. 2, pp. 480-486, 1993.

[14] Chitti B., et al. "Analysis of SDFT based phase detection system for grid synchronization of distributed generation systems." Engineering Science and Technology, an International Journal. Vol. 17, no 4, pp.0 270-278, 2014.

[15] Singh K. M. "Simultaneous Estimation of Moving-Vibration Parameters by Sliding Goertzel Algorithm in PLL Technique." IEEE Transactions on Instrumentation and Measurement. Vol. 68, no. 2, pp. 334-343, 2019.

\section{BIOGRAPHIES OF AUTHORS}

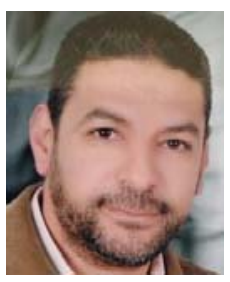

Osama M. Arafa was born in Tanta - EGYPT on December 25, 1965. He graduated from the Electrical Power and Machines Department of the Faculty of Engineering-Minofiya University in 1990. He received M.Sc. and Ph.D. from the Faculty of Engineering of Cairo University in 1998 and 2004 respectively. From 2004 to 2011 he worked for several automation companies and is currently working as an associate-professor at the Electronics Research Institute -Egypt. His research interests include Power Electronics, Energy Conversion, Control of Electrical Drives and Renewable Energy Systems.

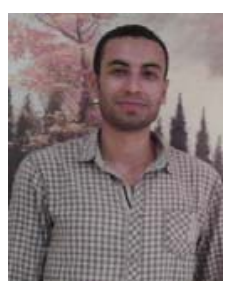

M. E. Abdallah was born in Shoubra, Egypt on January 3, 1989. He graduated from the Electrical Power and Machines Department of the Faculty of Engineering, Benha University on May 2011. His employment experience included the Egyptian Atomic Energy Authority and Electronic Research Institute. His specific fields of interest include Automatic Control and Renewable Energy. He is currently working as research assistant at Electronics Research Institute-Egypt.

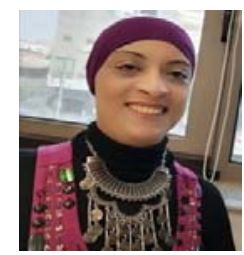

Ghada A. Abdel Aziz received the B.Sc and the M.Sc degrees in Electrical Engineering from Minofiya University, Egypt, in 2006, 2009, respectively, and the Ph.D. degree in Electrical Engineering, Cairo University, Egypt, in 2015. From 2006 to 2008, she was Teaching Assistant with several academic institutions in Egypt. Since 2009, she joined the Electronics Research Institute (ERI) in Egypt as a research assistant. From 2015 to present she is a researcher at ERI. Her current research interests include control of electrical machines, power electronics in sustainable energy systems, fault-tolerant control, and fault-tolerant design of electric machine and power electronics system. 